Klaus Miesenberger

Christian Bühler

Petr Penaz (Eds.)

\title{
Computers Helping People with Special Needs
}

15th International Conference, ICCHP 2016 Linz, Austria, July 13-15, 2016 Proceedings, Part II

Part II

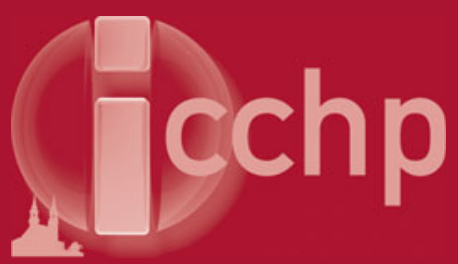

Springer 


\section{Lecture Notes in Computer Science}

Commenced Publication in 1973

Founding and Former Series Editors:

Gerhard Goos, Juris Hartmanis, and Jan van Leeuwen

\section{Editorial Board}

David Hutchison

Lancaster University, Lancaster, UK

Takeo Kanade

Carnegie Mellon University, Pittsburgh, PA, USA

Josef Kittler

University of Surrey, Guildford, UK

Jon M. Kleinberg

Cornell University, Ithaca, NY, USA

Friedemann Mattern

ETH Zurich, Zürich, Switzerland

John C. Mitchell

Stanford University, Stanford, CA, USA

Moni Naor

Weizmann Institute of Science, Rehovot, Israel

C. Pandu Rangan

Indian Institute of Technology, Madras, India

Bernhard Steffen

TU Dortmund University, Dortmund, Germany

Demetri Terzopoulos

University of California, Los Angeles, CA, USA

Doug Tygar

University of California, Berkeley, CA, USA

Gerhard Weikum

Max Planck Institute for Informatics, Saarbrücken, Germany 
More information about this series at http://www.springer.com/series/7409 
Klaus Miesenberger · Christian Bühler Petr Penaz (Eds.)

\section{Computers Helping People with Special Needs}

15th International Conference, ICCHP 2016

Linz, Austria, July 13-15, 2016

Proceedings, Part II

黛 Springer 


\section{Editors}

Klaus Miesenberger

Institute Integriert Studieren

Universität Linz

Linz

Austria

Christian Bühler

Rehabilitationswissenschaften

Technische Universität Dortmund

Dortmund

Germany

\author{
Petr Penaz \\ Masaryk University \\ Brno \\ Czech Republic
}

ISSN 0302-9743

ISSN 1611-3349 (electronic)

Lecture Notes in Computer Science

ISBN 978-3-319-41266-5 ISBN 978-3-319-41267-2 (eBook)

DOI 10.1007/978-3-319-41267-2

Library of Congress Control Number: 2016943068

LNCS Sublibrary: SL3 - Information Systems and Applications, incl. Internet/Web, and HCI

(C) Springer International Publishing Switzerland 2016

This work is subject to copyright. All rights are reserved by the Publisher, whether the whole or part of the material is concerned, specifically the rights of translation, reprinting, reuse of illustrations, recitation, broadcasting, reproduction on microfilms or in any other physical way, and transmission or information storage and retrieval, electronic adaptation, computer software, or by similar or dissimilar methodology now known or hereafter developed.

The use of general descriptive names, registered names, trademarks, service marks, etc. in this publication does not imply, even in the absence of a specific statement, that such names are exempt from the relevant protective laws and regulations and therefore free for general use.

The publisher, the authors and the editors are safe to assume that the advice and information in this book are believed to be true and accurate at the date of publication. Neither the publisher nor the authors or the editors give a warranty, express or implied, with respect to the material contained herein or for any errors or omissions that may have been made.

Printed on acid-free paper

This Springer imprint is published by Springer Nature

The registered company is Springer International Publishing AG Switzerland 


\section{Contents - Part II}

\section{Environmental Sensing Technologies for Visual Impairment}

Environmental Sensing Technologies for Visual Impairment: Introduction to the Special Thematic Session . . . . . . . . . . . . . . . . .

Roberto Manduchi and James Coughlan

Ball Course Detection Function for the Blind Bowling Support System

Using a Depth Sensor . . . . . . . . . . . . . . . . . . . .

Makoto Kobayashi

Catching the Right Bus - Improvement of Vehicle Communication with Bluetooth Low Energy for Visually Impaired and Blind People . . . . . . . . . . Elmar Krainz, Werner Bischof, Markus Dornhofer, and Johannes Feiner

Navi Rando: GPS-IMU Smart Phone Application for Helping Visually Impaired People Practicing Hiking

Jesus Zegarra Flores, Laurence Rasseneur, Clément Gass, and René Farcy

Scene Text Detection and Tracking for Wearable Text-to-Speech

Translation Camera . . . . . . . . . . . . . . . . . . . . . . .

Hideaki Goto and Kunqi Liu

Zebra Crossing Detection from Aerial Imagery Across Countries . . . . . . . . .

Daniel Koester, Björn Lunt, and Rainer Stiefelhagen

Sound of Vision - 3D Scene Reconstruction from Stereo Vision in an Electronic Travel Aid for the Visually Impaired .

Mateusz Owczarek, Piotr Skulimowski, and Pawel Strumillo

Experiments with a Public Transit Assistant for Blind Passengers . . . . . . . . . German Flores and Roberto Manduchi

\section{Tactile Graphics and Models for Blind People and Recognition of Shapes by Touch}

Electromagnetic Microactuator-Array Based Virtual Tactile Display. . . . . . . . Zoltan Szabo and Eniko T. Enikov

Empowering Low-Vision Rehabilitation Professionals with "Do-It-Yourself" Methods . . . . . . . . . . . . . . . . . . . . . 
Expansion Characteristic of Tactile Symbols on Swell Paper: Effects of Heat Setting, Position and Area of Tactile Symbols. . . . . . . . . . . . . .

Takahiro Hashimoto and Tetsuya Watanabe

Tactile Identification of Embossed Raised Lines and Raised Squares with Variable Dot Elevation by Persons Who Are Blind . . . . . . . . . . . .

Georgios Kouroupetroglou, Aineias Martos, Nikolaos Papandreou,

Konstantinos Papadopoulos, Vassilios Argyropoulous,

and Georgios D. Sideridis

Early Stimulation with Tactile Devices of Visually Impaired Children . . . . . .

María Visitación Hurtado Torres, María Luisa Rodríguez Almendros,

María José Rodríguez Fórtiz, Carlos Rodríguez Domínguez,

and María Bermúdez-Edo

Experimenting with Tactile Sense and Kinesthetic Sense Assisting System for Blind Education. . . . . . . . . . . . . . . . . . . . .

Junji Onishi, Tadahiro Sakai, Msatsugu Sakajiri, Akihiro Ogata,

Takahiro Miura, Takuya Handa, Nobuyuki Hiruma, Toshihiro Shimizu, and Tsukasa Ono

Locating Widgets in Different Tactile Information Visualizations . . . . . . . . Denise Prescher and Gerhard Weber

A Concept for Re-useable Interactive Tactile Reliefs . . . . . . . . . . . . . .

Andreas Reichinger, Anton Fuhrmann, Stefan Maierhofer, and Werner Purgathofer

Three-Dimensional Models of Earth for Tactile Learning . . . . . . . . . . . . .

Yoshinori Teshima, Yasunari Watanabe, Yohsuke Hosoya, Kazuma Sakai, Tsukasa Nakano, Akiko Tanaka, Toshiaki Aomatsu, Tatsuyoshi Tanji, Kenji Yamazawa, Yuji Ikegami, Mamoru Fujiyoshi, Susumu Oouchi, and Takeshi Kaneko

\section{Tactile Maps and Map Data for Orientation and Mobility}

Augmented Reality Tactile Map with Hand Gesture Recognition. . . . . . . . . . . Ryosuke Ichikari, Tenshi Yanagimachi, and Takeshi Kurata

Blind Friendly Maps: Tactile Maps for the Blind as a Part of the Public Map Portal (Mapy.cz) . . . . . . . . . . . . . . . . . . . . . . .

Petr Červenka, Karel Břinda, Michaela Hanousková, Petr Hofman, and Radek Seifert

BlindWeb Maps - An Interactive Web Service for the Selection and Generation of Personalized Audio-Tactile Maps . . . . . . . . . . . . . .

Timo Götzelmann and Laura Eichler 
CapMaps: Capacitive Sensing 3D Printed Audio-Tactile Maps . . . . . . . . . . Timo Götzelmann

Empirical Study on Quality and Effectiveness of Tactile Maps

Using HaptOSM System . . . . . . . . . . . . . . . . . . .

Daniel Hänßgen, Nils Waldt, and Gerhard Weber

Specification of Symbols Used in Audio-Tactile Maps for Individuals with Blindness ...........................

Konstantinos Papadopoulos, Konstantinos Charitakis, Eleni Koustriava, Lefkothea Kartasidou, Efstratios Stylianidis, Georgios Kouroupetroglou, Suad Sakalli Gumus, Karin Müller, and Engin Yilmaz

User Requirements Regarding Information Included in Audio-Tactile Maps for Individuals with Blindness . . . . . . . . . . . . . . . .

Konstantinos Papadopoulos, Konstantinos Charitakis,

Lefkothea Kartasidou, Georgios Kouroupetroglou, Suad Sakalli Gumus, Efstratios Stylianidis, Rainer Stiefelhagen, Karin Müller, Engin Yilmaz, Gerhard Jaworek, Christos Polimeras, Utku Sayin, Nikolaos Oikonomidis, and Nikolaos Lithoxopoulos

\section{Mobility Support for Blind and Partially Sighted People}

Obstacle Detection and Avoidance for the Visually Impaired in Indoors

Environments Using Google's Project Tango Device . . . . . . . . . . . . . . . .

Rabia Jafri and Marwa Mahmoud Khan

System Supporting Independent Walking of the Visually Impaired . . . . . . . .

Mitsuki Nishikiri, Takehiro Sakai, Hiroaki Kudo, Tetsuya Matsumoto, Yoshinori Takeuchi, and Noboru Ohnishi

Path Planning for a Universal Indoor Navigation System . . . . . . . . . . .

Elie Kahale, Pierre-Charles Hanse, Valéria Destin, Gérard Uzan, and Jaime Lopez-Krahe

Supporting Pedestrians with Visual Impairment During Road Crossing:

A Mobile Application for Traffic Lights Detection . . . . . . . . . . . .

Sergio Mascetti, Dragan Ahmetovic, Andrea Gerino, Cristian Bernareggi, Mario Busso, and Alessandro Rizzi

Sound of Vision - Spatial Audio Output and Sonification Approaches . . . . . . Michal Bujacz, Karol Kropidlowski, Gabriel Ivanica, Alin Moldoveanu, Charalampos Saitis, Adam Csapo, György Wersenyi, Simone Spagnol, Omar I. Johannesson, Runar Unnthorsson, Mikolai Rotnicki, and Piotr Witek 


\section{The Use of Mobile Devices by Individuals with Special Needs} as an Assistive Tool

Mobile Learning for People with Special Needs: Introduction to the Special

Thematic Session ........................... 212

Linda Chmiliar

Parents' and Teachers' Perspectives on Using IPads with Students with

Developmental Disabilities: Applications for Universal Design for Learning . . . Therese M. Cumming and Iva Strnadová

The iPad Helping Preschool Children with Disabilities

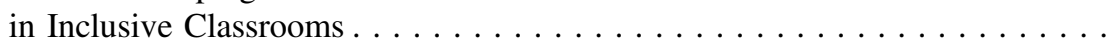

Linda Chmiliar

Easy Access to Social Media: Introducing the Mediata-App . . . . . . . . . . .

Christian Bühler, Susanne Dirks, and Annika Nietzio

A Tool to Improve Visual Attention and the Acquisition of Meaning for Low-Functioning People . . . . . . . . . . . . . . . . . . . . .

María Luisa Rodríguez Almendros, Marcelino Cabrera Cuevas,

Carlos Rodríguez Domínguez, Tomás Ruiz López, María Bermúdez-Edo, and María José Rodríguez Fórtiz.

\section{Mobility Support for People with Motor and Cognitive Disabilities}

A Mobile Travel Companion Based on Open Accessibility Data. . . . . . . . .

Christophe Ponsard, Fabrice Estiévenart, Valery Ramon, Alexandre Rosati, Emilie Goffin, Vincent Snoeck, and Stéphanie Hermans

Mobility Support for People with Dementia . . . . . . . . . . . . . . . Reinhard Koutny and Klaus Miesenberger

Community Engagement Strategies for Crowdsourcing Accessibility

Information: Paper, Wheelmap-Tags and Mapillary-Walks . . . . . . . . . . .

Christian Voigt, Susanne Dobner, Mireia Ferri, Stefan Hahmann, and Karsten Gareis

Sharing Real-World Accessibility Conditions Using a Smartphone

Application by a Volunteer Group. . . . . . . . . . . . . . . . . . . .

Takahiro Miura, Ken-ichiro Yabu, Takeshi Noro, Tomoko Segawa,

Kei Kataoka, Akihito Nishimuta, Masaya Sanmonji, Atsushi Hiyama, Michitaka Hirose, and Tohru Ifukube

Development and Evaluation of Navigation System with Voice and

Vibration Output Specialized for Persons with Higher Brain Dysfunction. . . .

Akihiko Hanafusa, Tsuyoshi Nojiri, and Tsuyoshi Nakayama 
SIMON: Integration of ICT Solutions for Mobility and Parking . . . . . . . . .

Alberto Ferreras, José Solaz, Eva María Muñoz, Manuel Serrano, Antonio Marqués, Amparo López, and José Laparra

\section{Towards e-Inclusion for People with Intellectual Disabilities}

Towards e-Inclusion for People with Intellectual Disabilities: Introduction to the Special Thematic Session . . . . . . . . . . . . . . . . Cordula Edler

Criteria of Barrier-Free Websites for the Vocational Participation of People with Cognitive Disabilities. An Expert Survey Within the Project

"Online-Dabei". . . . . . . . . . . . . . . . . . . . . . . . . . .

Elena Brinkmann, Lena Bergs, Marie Heide, and Mathilde Niehaus

Easy Reader - or the Importance of Being Understood . . . . . . . . . . . . .

Peter Heumader, Cordula Edler, Klaus Miesenberger, and Andrea Petz

Potentials of Digital Technology for Participation of Special Needs

Children in Kindergarten . . . . . . . . . . . . . . . . . .

Isabel Zorn, Jennifer Justino, Alexandra Schneider,

and Jennifer Schönenberg

Reordering Symbols: A Step Towards a Symbol to Arabic Text Translator. . . .

Lama Alzaben, Mike Wald, and E.A. Draffan

SAMi: An Accessible Web Application Solution for Video Search for People with Intellectual Disabilities . . . . . . . . . . . . . .

Tânia Rocha, Hugo Paredes, João Barroso, and Maximino Bessa

Target Group Questionnaire in the "ISG for Competence" Project. . . . . . . . .

Szilvia Paxian, Veronika Szücs, Shervin Shirmohhamadi, Boris Aberšek,

Andrean Lazarov, Karel Van Isacker, and Cecilia Sik-Lanyi

The Development and Evaluation of an Assistance System for Manual Order Picking - Called Pick-by-Projection - with Employees with Cognitive Disabilities . . . . . . . . . . . . . . . . . . . .

Andreas Baechler, Liane Baechler, Sven Autenrieth, Peter Kurtz,

Georg Kruell, Thomas Hoerz, and Thomas Heidenreich

The Use and Impact of an Assistance System for Supporting Participation in Employment for Individuals with Cognitive Disabilities . . . . . . . . . . . .

Liane Baechler, Andreas Baechler, Markus Funk, Sven Autenrieth, Georg Kruell, Thomas Hoerz, and Thomas Heidenreich 


\section{AT and Inclusion of People with Autism or Dyslexia}

Internal Validity in Experiments for Typefaces for People with Dyslexia:

A Literature Review . . . . . . . . . . . . . . . . . . . . . . .

Trenton Schulz

Characterization of Programmers with Dyslexia . . . . . . . . . . . José L. Fuertes, Luis F. González, and Loïc Martínez.

What Technology for Autism Needs to be Invented? Idea Generation from the Autism Community via the ASCmeI.T. App . . . . . . . . . . . . .

Sarah Parsons, Nicola Yuill, Judith Good, Mark Brosnan, Lisa Austin, Clarence Singleton, Benoît Bossavit, and Barnabear

Using Mind Mapping Software to Initiate Writing and Organizing Ideas for Students with SLD and ADHD . . . . . . . . . . . . Betty Shrieber

Data Quality as a Bottleneck in Developing a Social-Serious-Game-Based Multi-modal System for Early Screening for 'High Functioning' Cases of Autism Spectrum Condition . . . . . . . . . . . . . . . . . . . . Miklos Gyori, Zsófia Borsos, Krisztina Stefanik, and Judit Csákvári

Interpersonal Distance and Face-to-face Behavior During Therapeutic Activities for Children with ASD . . . . . . . . . . . . . . . . Airi Tsuji, Soichiro Matsuda, and Kenji Suzuki

\section{AT and Inclusion of Deaf and Hard of Hearing People}

Support System for Lecture Captioning Using Keyword Detection by Automatic Speech Recognition. . . . . . . . . . . . . . . . . .

Naofumi Ikeda, Yoshinori Takeuchi, Tetsuya Matsumoto, Hiroaki Kudo, and Noboru Ohnishi

Compensating Cocktail Party Noise with Binaural Spatial Segregation on a Novel Device Targeting Partial Hearing Loss . . . . . . . . . . . . . . . 384 Luca Giuliani, Sara Sansalone, Stefania Repetto, Federico Traverso, and Luca Brayda

CoUnSiL: Collaborative Universe for Remote Interpreting of Sign

Language in Higher Education . . . . . . . . . . . . . . . . . .

Vit Rusňák, Pavel Troubil, Svatoslav Ondra, Tomáš Sklenák, Desana Daxnerová, Eva Hladká, Pavel Kajaba, Jaromír Kala, Matej Minárik, Peter Novák, and Christoph Damm

Classifiers in Arab Gloss Annotation System for Arabic Sign Language. . . . . 400 Nadia Aouiti and Mohamed Jemni 
Image-Based Approach for Generating Facial Expressions . . . . . . . . . . . .

Ibtissem Talbi, Oussama El Ghoul, and Mohamed Jemni

Developing eLecture Materials for Hearing Impaired Students and Researchers: Designing Multiple-Video Programs and Usability Assessment.

Ritsuko Kikusawa, Motoko Okumoto, Takuya Kubo, and Laura Rodrigo

Effect of Vibration on Listening Sound for a Person with Hearing Loss. . . . . Junichi Kanebako, Toshimasa Yamanaka, and Miki Namatame

Designing a Collaborative Interaction Experience for a Puppet Show System for Hearing-Impaired Children . . . . . . . . . . . . . . . . . Ryohei Egusa, Tsugunosuke Sakai, Haruya Tamaki, Fusako Kusunoki, Miki Namatame, Hiroshi Mizoguchi, and Shigenori Inagaki

SingleScreenFocus for Deaf and Hard of Hearing Students. . . . . . . . . . . Raja S. Kushalnagar, Poorna Kushalnagar, and Fadi Haddad

A Web Application for Geolocalized Signs in Synthesized Swiss German Sign Language . . . . . . . . . . . . . . . . . . . . . . . . . . . 438 Anna Jancso, Xi Rao, Johannes Grä̈n, and Sarah Ebling

\section{Accessible Computer Input}

Evaluation of a Mobile Head-Tracker Interface for Accessibility . . . . . . . . . Maria Francesca Roig-Maimó, Cristina Manresa-Yee, Javier Varona, and I. Scott MacKenzie

Replacement of the Standard Computer Keyboard and Mouse by Eye Blinks. . . . . . . . . . . . . . . . . . . . . . . Muhammad Bilal Saif and Torsten Felzer

SWIFT: A Short Word Solution for Fast Typing. . . . . . . . . . . . . . . 464 Philippe Roussille and Mathieu Raynal

An Empirical Evaluation of MoonTouch: A Soft Keyboard for Visually Impaired People . . . . . . . . . . . . . . . . . . . . . . . . Saber Heni, Wajih Abdallah, Dominique Archambault, Gérard Uzan, and Mohamed Salim Bouhlel

Zoning-Based Gesture Recognition to Enable a Mobile Lorm Trainer . . . . . . Michael Schmidt, Cathleen Bank, and Gerhard Weber

HandiMathKey: Mathematical Keyboard for Disabled Person . . . . . . . . . . . Elodie Bertrand, Damien Sauzin, Frédéric Vella, Nathalie Dubus, and Nadine Vigouroux 
A Study of an Intention Communication Assisting System

Using Eye Movement . . . . . . . . . . . . . . . . . . . .

Shogo Matsuno, Yuta Ito, Naoaki Itakura, Tota Mizuno, and Kazuyuki Mito

A Review of Computer-Based Gesture Interaction Methods for Supporting

Disabled People with Special Needs . . . . . . . . . . . . . .

Chutisant Kerdvibulvech

Optimizing Vocabulary Modeling for Dysarthric Speech Recognition . . . . . . Minsoo Na and Minhwa Chung

Comparison of Two Methods to Control the Mouse Using a Keypad. . . . . . . Torsten Felzer, Ian Scott MacKenzie, and John Magee

\section{AT and Rehabilitation for People with Motor and Mobility Disabilities}

Kalman-Based Approach to Bladder Volume Estimation for People with Neurogenic Dysfunction of the Urinary Bladder. . . . . . . . . . . . . . Alessandro Palla, Claudio Crema, Luca Fanucci, and Paolo Bellagente

Sound Feedback Assessment for Upper Limb Rehabilitation Using a Multimodal Guidance System . . . . . . . . . . . . . . . . . . Mario Covarrubias Rodriguez, Mauro Rossini, Giandomenico Caruso, Gianluca Samali, Chiara Giovanzana, Franco Molteni, and Monica Bordegoni

Personal Mobility Vehicle for Assisting Short-Range Transportation . . . . . . . Yoshiyuki Takahashi

Multimodal Sequential Modeling and Recognition of Human Activities . . . . . Mouna Selmi and Mounîm A. El-Yacoubi

Android Games for Developing Fine Coordination of Movement Skills . . . . . Tibor Guzsvinecz, Veronika Szücs, Szilvia Paxian, and Cecilia Sik Lanyi

\section{HCI, AT and ICT for Blind and Partially Sighted People}

Extending the Technology Enhanced Accessible Interaction Framework

Method for Thai Visually Impaired People. . . . . . . . . . . . . . . . .

Kewalin Angkananon and Mike Wald

Types of Problems Elicited by Verbal Protocols for Blind

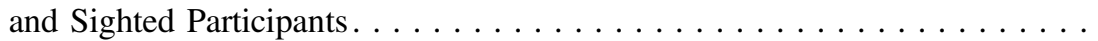
Andreas Savva, Helen Petrie, and Christopher Power

Multimodal Attention Stimulator 568 Andrzej Czyzewski, Bozena Kostek, and Lukasz Kosikowski 
Assessing Braille Input Efficiency on Mobile Devices . .

Norbert Márkus, Szabolcs Malik, and András Arató

Smart Glasses for the Visually Impaired People Esra Ali Hassan and Tong Boon Tang

EasyTrans: Accessible Translation System for Blind Translators . . . . . . . . 583 Dina Al-Bassam, Hessah Alotaibi, Samira Alotaibi, and Hend S. Al-Khalifa

An Accessible Environment to Integrate Blind Participants into Brainstorming Sessions: User Studies . . . . . . . . . . . . . . Stephan Pölzer, Andreas Kunz, Ali Alavi, and Klaus Miesenberger

Elements of Adaptation in Ambient User Interfaces. .

Laura Burzagli, Fabio Gori, Paolo Baronti, Marco Billi, and Pier Luigi Emiliani 


\section{Contents - Part I}

\section{Art Karshmer Lectures in Access to Mathematics, Science and Engineering}

Art Karshmer Lectures in Access to Mathematics, Science and Engineering:

Introduction to the Special Thematic Session . . . . . . . . . . . . . . .

Dominique Archambault, Katsuhito Yamaguchi,

Georgios Kouroupetroglou, and Klaus Miesenberger

Artificial Neural Networks and Fuzzy Logic for Recognizing Alphabet

Characters and Mathematical Symbols . . . . . . . . . . . . . . . .

Giuseppe Airò Farulla, Tiziana Armano, Anna Capietto, Nadir Murru, and Rosaria Rossini

Braille Math Extension to RoboBraille: A Universal Software Solution for Converting Math into Braille. . . . . . . . . . . . . . . . . . .

Vlad Paul Cosma, Tanja Stevns, and Lars Ballieu Christensen

Analysis of Implicit Didactics in Math Schoolbooks for Interactive

Non-visual User Interface Development . . . . . . . . . . . . . . . . . . .

Prajaks Jitngernmadan, Andrea Petz, Bernhard Stöger, and Klaus Miesenberger

An Evaluation Methodology of Math-to-Speech in Non-English DAISY

Digital Talking Books . . . . . . . . . . . . . . . . . . . .

Paraskevi Riga, Georgios Kouroupetroglou, and Polyxeni-Parthena Ioannidou

Recognition of E-Born PDF Including Mathematical Formulas . . . . . . . . . . Masakazu Suzuki and Katsuhito Yamaguchi

Polyfilling Accessible Chemistry Diagrams. . . . . . . . . . . . . . Volker Sorge

Tangible Programming Gimmick Using RFID Systems Considering the Use of Visually Impairments . . . . . . . . . . . . . . . . . . . . . . . . . . . .

Tatsuo Motoyoshi, Naoki Tetsumura, Hiroyuki Masuta,

Ken'ihci Koyanagi, Toru Oshima, and Hiroshi Kawakami

A Study of Speech Versus Braille and Large Print

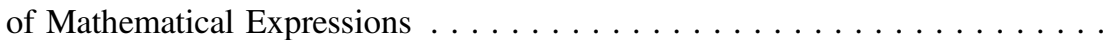

Neil Soiffer

Guidelines for Accessible Textual UML Modeling Notations . . . . . . . . . . .

Vanessa Petrausch, Stephan Seifermann, and Karin Müller 


\section{Technology for Inclusion and Participation}

Technology for Inclusion and Participation: Introduction to the Special

Thematic Session . . . . . . . . . . . . . . . . . . . .

Christian Bühler and Bastian Pelka

Co-creating an Online TimeBank for Inclusive Research . . . . . . . . . . . .

Sarah Parsons, Andrew Power, Melanie Nind, Ken Meacham,

Clare Hooper, Anne Collis, Mal Cansdale, and Alan Armstrong

Implementing UNCRPD - Strategies of Accessibility Promotion

and Assistive Technology Transfer in North Rhine-Westphalia . . . . . . . . . .

Michael Hubert, Christian Bühler, and Wolfgang Schmitz.

Developing a Technology-Based Speech Intervention for Acquired

Dysarthria: A Psychological Approach. . . . . . . . . . . . . . . . .

Ute Ritterfeld, Juliane Muehlhaus, Hendrike Frieg, and Kerstin Bilda

Re-thinking Assistive Technology Service Delivery Models in the Light

of the UN Convention $\ldots \ldots \ldots \ldots \ldots \ldots \ldots \ldots \ldots \ldots \ldots$

Renzo Andrich

Standards Related to eAccessibility and eInclusion: Dimensions of Interoperability Based on Standards with Respect to eAccessibility

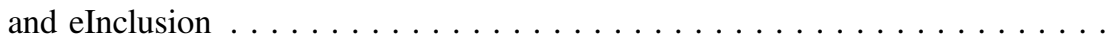

Christian Galinski and Blanca Stella Giraldo Perez

Fostering the Development of Inclusively Minded Engineers . . . . . . . . . .

Seiichiro Miura, Naohiro Hayashi, Sakiko Ogoshi, Hitoshi Nishi,

Takashi Yoshioka, Yutaka Yamaguchi, and Yasuhiro Ogoshi

Cloud Computing in European Schools - The Impact

on Inclusive Education.

Ingo Karl Bosse

Methodology for Universal Design of ITs; Epistemologies

Among Norwegian Experts $\ldots \ldots \ldots \ldots \ldots \ldots \ldots$

Miriam Eileen Nes Begnum

Using ICT and Quality of Life: Comparing Persons with

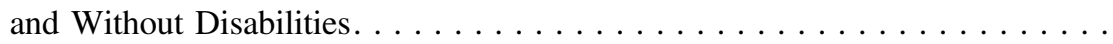

Djordje M. Kadijevich, Gordana Odovic, and Dejan Maslikovic

The Use of Working Prototypes for Participatory Design with People with Disabilities

Brandon Haworth, Muhammad Usman, Melanie Baljko,

and Foad Hamidi 
Supported Employment - Electronic Job-Coach (EJO). . .

Carsten Brausch, Christian Bühler, Andreas Feldmann, and Miriam Padberg

\section{Mobile Apps and Platforms}

Mobile Apps and Platforms: Introduction to the Special Thematic Session . . . Marion Hersh and Alireza Darvishy

Can Visually Impaired Smartphone Users Correctly Manipulate Tiny

Screen Keyboards Under a Screen Reader Condition? . . . . . . . . . . . . . .

Takashi Ohashi, Takahiro Miura, Masatsugu Sakajiri, Junji Onishi, and Tsukasa Ono

Working with Frail, Elderly EVA Users to Determine Ways to Support Agency in Single Switch Connectivity with Mobile Technologies . . . . . . . .

Lawrence Kwok, Margot Whitfield, Sander Fels-Leung, Mauricio Meza, and Deborah Fels

Towards a Natural User Interface to Support People with Visual

Impairments in Detecting Colors.....................

Sergio Mascetti, Chiara Rossetti, Andrea Gerino, Cristian Bernareggi,

Lorenzo Picinali, and Alessandro Rizzi

Cognitive Accessibility to Mobile ICT. . . . . . . . . . . . . . .

Martin Böcker, Nikolaos Floratos, Loïc Martínez, Mike Pluke,

Bruno Von Niman, and Gill Whitney

What Do Low-Vision Users Really Want from Smart Glasses? Faces,

Text and Perhaps No Glasses at All . . . . . . . . . . . . .

Frode Eika Sandnes

\section{Accessibility of Web and Graphics}

Image Processing and Pattern Recognition Tools for the Automatic

Image Transcription. . . . . . . . . . . . . . . . . . .

Zehira Haddad, Yong Chen, and Jaime Lopez Krahe

Generating Image Descriptions for SmartArts . . . . . . . . . . . . . . . . Jens Voegler, Julia Krause, and Gerhard Weber

Mobile Interactive Image Sonification for the Blind.

Torsten Wörtwein, Boris Schauerte, Karin Müller, and Rainer Stiefelhagen

Online Graphics for the Blind: Intermediate Format Generation

for Graphic Categories. . . . . . . . . . . . . . . . . . . .

Roopa Bose and Helmut Jürgensen 
LiveDescribe Web Redefining What and How Entertainment Content

Can Be Accessible to Blind and Low Vision Audiences. . . . . . . . . . . . . . Margot Whitfield, Raza Mir Ali, and Deborah Fels

Insights into Internet Privacy for Visually Impaired and Blind People . . . . . . Georg Regal, Elke Mattheiss, Marc Busch, and Manfred Tscheligi

Understanding How People with Cerebral Palsy Interact with the Web 2.0. . . Leticia Seixas Pereira and Dominique Archambault

Developing a Framework for Localised Web Accessibility Guidelines for University Websites in Saudi Arabia . . . . . . . . . . . . . . . . Asmaa Alayed, Mike Wald, and E.A. Draffan

Towards Accessible Innovative Assessment Items . . . . . . . . . . . . . . . . . .

Eric G. Hansen, Carlos Cavalie, Teresa King, Mark T. Hakkinen, Jason J. White, and Jennifer Grant

Web Page Clustering for More Efficient Website Accessibility Evaluations. . . . Justyna Mucha, Mikael Snaprud, and Annika Nietzio

\section{Ambient Assisted Living (AAL) for Aging and Disability}

SEAMPAT: An ICT Platform for Medication Reconciliation with Active Patient Involvement. . . . . . . . . . . . . . . . . . . . . . . . . .

Valéry Ramon, Ravi Ramdoyal, Sophie Marien, Arnaud Michot, Jimmy Nsenga, Gustavo Ospina, Fabian Steels, Quentin Boucher, Delphine Legrand, Christine Burnet, Perrine Vanmerbeek, and Anne Spinewine

BRELOMATE - A Distributed, Multi-device Platform for Online Information, Communication and Gaming Services Among the Elderly . . . . . Jakob Doppler, Sabine Sommer, Christian Gradl, and Gernot Rottermanner

Electronic Medication Dispensers Finding the Right Users - A Pilot Study in a Norwegian Municipality Home Care Service . . . . . . . . . . . . . . 281 Ingrid S. Svagård and Elin S. Boysen

Multidimensional Observation Methodology for the Elderly in an Ambient Digital Environment . . . . . . . . . . . . . . . . . . . . . Adrien Van den Bossche, Eric Campo, Jenny Duchier, Elizabeth Bougeois, Mathilde Blanc Machado, Thierry Val, Frédéric Vella, and Nadine Vigouroux 
An Exploratory Framework Assessing Intrinsic and Extrinsic

Motivators Related to Mobile Device Applications and Attributes

for the Canadian Seniors . . . . . . . . . . . . . . . . . . . . . . . .

Susan E. Reid, Bessam Abdulrazak, and Monica Alas

One Size Does Not Fit All: Design and Implementation Considerations

When Introducing Touch Based Infotainment Systems to Nursing

Home Residents . . . . . . . . . . . . . . . . . . . . . . . . .

Øystein Dale, Elin Sundby Boysen, and Ingrid Svagård

Support Services for Informal Caregivers: Survey with Providers in Austria . . . Susanne Hensely-Schinkinger

AAL-Case Management: A Key to Successful Service Provision. Sabine Katzmaier

What People with Dementia Want: Designing MARIO an Acceptable

Robot Companion . . . . . . . . . . . . . . . . . . . . . . . . . .

Dympna Casey, Heike Felzmann, Geoff Pegman,

Christos Kouroupetroglou, Kathy Murphy, Adamantios Koumpis, and Sally Whelan

The EnrichMe Project: A Robotic Solution for Independence and Active Aging of Elderly People with MCI . . . . . . . . . . . . . . . .

Claudia Salatino, Valerio Gower, Meftah Ghrissi, Adriana Tapus, Katarzyna Wieczorowska-Tobis, Aleksandra Suwalska, Paolo Barattini, Roberto Rosso, Giulia Munaro, Nicola Bellotto, and Herjan van den Heuvel

Walking Aid Identification Using Wearables . . . . . . . . . . . . . .

Thomas Moder, Karin Wisiol, and Manfred Wieser

How Can We Develop AAC for Dementia? . . . . . . . . . . . . . .

John L. Arnott and Norman Alm

\section{The Impact of PDF/UA on Accessible PDF}

The Impact of PDF/UA on Accessible PDF: Introduction to the Special Thematic Session Olaf Drümmer

Are PDFs an Accessible Solution?

Gian Wild and Daniel Craddock

A Simple Viewer and Editor of Sound-Embedded PDF for Elementary School Students with Print Disabilities. . . . . . . . . . . . . . . . . . .

Yoshiaki Tani, Takuya Takaira, and Akio Fujiyoshi 
Automatic Paragraph Detection for Accessible PDF Documents . . . . . . . . . Alireza Darvishy, Mark Nevill, and Hans-Peter Hutter

PDF/UA and the User Experience Survey Karen McCall

\section{Standards, Tools and Procedures in Accessible eBook Production}

Standards, Tools and Procedures in Accessible eBook Production: Introduction to the Special Thematic Session . . . . . . . . . . . . . . . . Reinhard Ruemer, Georgios Kouroupetroglou, Tomas Murillo-Morales, and Klaus Miesenberger

Development of a Unified Production System for Various Types of Accessible Textbooks ......................... Takuya Takaira, Yoshiaki Tani, and Akio Fujiyoshi

RISE eBooks: Leveraging Off-the-Shelf Software Components in Support of Deaf Literacy . . . . . . . . . . . . . . . . . . . . . . . .

Riley Collins, Gene Mirus, and Donna Jo Napoli

A LaTeX to Braille Conversion Tool for Creating Accessible Schoolbooks in Austria. . . . . . . . . . . . . . . . . . . . . . . . . .

Tomás Murillo-Morales, Klaus Miesenberger, and Reinhard Ruemer

Practical Evaluation of DaisyRings: Text-to-Speech-Based DAISY Content

Creation System . . . . . . . . . . . . . . . . . . . . . . . Kosei Fume, Yuka Kuroda, Taira Ashikawa, and Masahiro Morita

HERMOPHILOS: A Web-Based Information System for the Workflow Management and Delivery of Accessible eTextbooks. . . . . . . . . . . . . . Alexandros Pino, Georgios Kouroupetroglou, and Paraskevi Riga

Universally Accessible Figures . . . . . . . . . . . . . . . . . . John A. Gardner

\section{Accessible eLearning - eLearning for Accessibility/AT}

Accessible eLearning - eLearning for Accessibility/AT: Introduction to the Special Thematic Session . . . . . . . . . . . . . . . . . . . E.A. Draffan and Peter Heumader

A Personal Reflection on Developing a Digital Accessibility MOOC Compared to Developing a Traditional Course . . . . . . . . . . . . . Mike Wald 


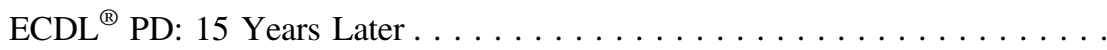

Andrea Petz and Klaus Miesenberger

How Accessible Are MOOCs to the Elderly?: A Case Study on a MOOC

Demo Course . . . . . . . . . . . . . . . . . . . . . . . . . . . . . .

Way Kiat Bong and Weiqin Chen

OLA! A Scenario-Based Approach to Enhance Open Learning

Through Accessibility . . . . . . . . . . . . . . . . . .

Tim Coughlan, Alejandro Rodriguez-Ascaso, Francisco Iniesto, and Anne Jelfs

Teaching Accessibility with Personas.

Claudia Loitsch, Gerhard Weber, and Jens Voegler

Learning Through Videos: Are Disabled Students

Using Good Note-Taking Strategies? .

Abi James, E.A. Draffan, and Mike Wald

\section{Inclusive Settings, Pedagogies and Approaches in ICT-Based Learning for Disabled and Non-disabled People}

Inclusive Settings, Pedagogies and Approaches in ICT Based Learning for

Disabled and Non-disabled People: Introduction to the Special Thematic Session. . . Marion Hersh

Designing Effective Learning Technologies for Autistic People. Marion Hersh

Efficacy of Tablets for Students with Learning Difficulties Studying Concepts in Geometry . . . . . . . . . . . . . . . . . . Betty Shrieber and David Eldar

Using Biometrics to Support Affective eLearning for Users with Special Needs

Ian Pitt, Tracey Mehigan, and Katie Crowley

Haptic Models of Arrays Through 3D Printing for Computer

Science Education.

Nicola Papazafiropulos, Luca Fanucci, Barbara Leporini,

Susanna Pelagatti, and Roberto Roncella

\section{Digital Games Accessibility}

Design of a Curriculum Framework for Raising Awareness of Game Accessibility . . . . . . . . . . . . . . . . . . 
Identifying Existing, Accessible Touchscreen Games for People

Living with Dementia . . . . . . . . . . . . . . . . .

Phil Joddrell, Alexandra Hernandez, and Arlene J. Astell

The Role of Small Robots in Designed Play Workshops in Centers

of Adults with Cerebral Palsy . . . . . . . . . . . . . . . . . . . .

Isabel M. Gómez, Rubén Rodríguez, Juan Jesús Otero, Manuel Merino, Alberto J. Molina, and Rafael Cabrera

How to Control a Mobile Game: A Comparison of Various Approaches for Visually Impaired People . . . . . . . . . . . . . . . . . . . .

Krzysztof Dobosz and Jakub Ptak

Training Working Memory in Elderly People with a Computer-Based Tool . . . Sandra Rute-Pérez, Carlos Rodríguez-Domínguez, María José Rodríguez-Fórtiz, María Visitación Hurtado-Torres, and Alfonso Caracuel

Audible Mapper \& ShadowRine: Development of Map Editor Using only Sound in Accessible Game for Blind Users, and Accessible Action RPG for Visually Impaired Gamers. . . . . . . . . . . . . . . . . . . . . . . . . Masaki Matsuo, Takahiro Miura, Masatsugu Sakajiri, Junji Onishi, and Tsukasa Ono

Memory Game and Special HCI Device in Stroke Therapy . . . . . . . . . . . Máté Godár, Veronika Szücs, and Cecilia Sik-Lanyi

\section{User Experience and Emotions for Accessibility (UEE4A)}

User Experience and Emotions for Accessibility: Introduction to the Special Thematic Session . . . . . . . . . . . . . . . . . . . . . . . Yehya Mohamad, Gaby Nordbrock, Henrike Suzuki, and Carlos Velasco

A Smart Clothe for ECG Monitoring of Children with Autism Spectrum Disorders . . . . . . . . . . . . . . . . . . . . . . Kanako Takahashi, Soichiro Matsuda, and Kenji Suzuki

Study on Elicitation and Detection of Emotional States with Disabled Users. . . 563 Yehya Mohamad, Gaby Nordbrock, Henrike Gappa, and Carlos Velasco

Usability Evaluation of Accessible Complex Graphs Dimitris Spiliotopoulos, Despoina Antonakaki, Sotiris Ioannidis, and Paraskevi Fragopoulou 


\title{
Optimizing Vocabulary Modeling for Dysarthric Speech Recognition
}

\author{
Minsoo $\mathrm{Na}^{1}$ and Minhwa Chung ${ }^{2(\bowtie)}$ \\ ${ }^{1}$ Interdisciplinary Program in Cognitive Science, Seoul National University, \\ Seoul, South Korea \\ dix39@snu.ac.kr \\ ${ }^{2}$ Department of Linguistics, Seoul National University, Seoul, South Korea \\ mchung@snu.ac.kr
}

\begin{abstract}
Imperfection in articulation of dysarthric speech results in the deterioration on the performance of speech recognition. In this paper, the effect of the articulating class of phonemes in the dysarthric speech recognition results is analyzed using generalized linear mixed models (GLMMs). The model with the features categorized according to the manner of articulation and the place of tongue is selected as the best one by the analysis. Recognition accuracy score for each word is predicted based on its pronunciation and the GLMM. The vocabulary optimized by selecting words with the maximum score shows a $16.4 \%$ relative error reduction in dysarthric speech recognition.
\end{abstract}

Keywords: Dysarthria $\cdot$ Vocabulary modeling $\cdot$ Speech recognition

\section{Introduction}

Dysarthria is a motor speech disorder caused by damage to the central or peripheral nervous system. As the error rate of recognition is significantly higher when dysarthric speakers use an ASR system developed for non-dysarthric speakers, researchers have focused on improving the models for recognition. The speech recognizer is composed of models such as acoustic model, pronunciation model, vocabulary and language model. The acoustic characteristic of dysarthric speech is analyzed and dysarthric speech is converted to be heard as normal speech [1]. The acoustic model is improved by using speaker adaptation or by using DNN [2]. Confusions in pronunciation are modeled as pronunciation variants or embedded to the search network [3]. In ASR-based applications developed for dysarthric speakers, recognition errors are reduced not only by improving the models but also by defining problems in the application of ASR with small vocabulary and simple-patterned utterances for lowering perplexity [4-6]. The voice keyboard [5] is an interface for inputting the mapped key by recognizing an isolated word utterance, which is a method for text input using small vocabulary isolated word recognition. A phonetic alphabet is set as the default vocabulary for the interface $[5,6]$. Customizing the vocabulary leads to a decrease in the errors in recognition [6], however, the customization is performed by trial and error. One of the characteristics of dysarthric speech is imperfection in articulation. The frequencies of articulation errors of phoneme and of class of phoneme are observed in 
dysarthric speech [7, 8]. For consonants, fricatives and affricates show higher error rates when classified by manner of articulation and alveolars show higher rates when classified by place of articulation and the error rate for monophthongs at extreme positions such as /i, ae, a/ is higher [7]. The difference between recognition accuracy by ASR system and score of intelligibility test by human rater is observed [9].

From previous researches, we assume that error rates in recognition depend on the class of the phoneme and the effect of the articulating the classes in recognition result rather than in the judgement of human raters is analyzed in order to estimate the recognition performance of each word based on its phoneme-level pronunciation. We analyze the effect of the articulating class of phoneme in dysarthric speech recognition result using the generalized linear mixed model (GLMM) and define the recognition score based on the analysis of optimizing the vocabulary for improving the ASR performance.

\section{Method}

In our voice keyboard, users enter text into the application by repeatedly uttering a recognition word for a target alphabet. Korean graphemes are composed of 19 consonants, 8 monophthongs, and 13 diphthongs. We exclude 5 consonants for fortis and 9 less frequent diphthongs from arrangement of the keys in the voice keyboard due to lack of data, which results in the keyboard having a total of 47 keys for 14 consonantal graphemes, 12 vowel graphemes and 21 control functions.

Two sets of isolated word corpus of Korean dysarthric speakers are used for analysis and evaluation [10]. The vocabulary of one corpus is composed of 173 words and the other is 500 words. Recording the word utterances is repeated at least twice for 13 speakers. One set among two sets of the utterance is used for the acoustic model adaptation, and the other is used for recognition test. The GMM-HMM models are trained and adapted by applying fMLLR and MAP methods [11]. The isolated word grammar for a total of 47 words in a vocabulary is made to define the search network. The number of phonemes for each class in the canonical pronunciation of the word for an utterance in the speech corpus is counted. Severity of the speaker and four types of counts according to criteria of classification of consonants and vowels are written to form the fixed effects of the generalized linear mixed model (GLMM). Speaker ID and word ID are also included as the random effects in the model. As a result, four GLMMs are built to predict the recognition accuracy: Place-Frontness model, Place-Height model, Manner-Frontness model and Manner-Height model. Among the models, the Manner-Frontness model minimizes the negative of log likelihood and the model size. The estimates of the model are shown in Table 1.

Fricative, Nasal, Front, Central, Back and Severity are significant $(p<0.05)$. Accuracy decreases as Severity and the number of Fricative and Lateral increases. Accuracy increases as the number of Nasal, Plosive, Affricate, Diphthongs and monophthongs increases.

The word set of the speech corpus includes three types of alphabet word lists whose words are selected based on their ease of articulation. Words in each alphabet word lists and 1-best words among control candidate set are combined to yield three kinds of 
Table 1. Estimate of the Manner-Frontness model.

\begin{tabular}{l|l|l|l|l|l|l}
\hline Effects & Intercept & Plosive & Fricative & Affricate & Nasal & Lateral \\
\hline Estimate & 3.96 & 0.04 & -0.26 & 0.03 & 0.20 & -0.04 \\
\hline Sign. & $* * *$ & & $*$ & & $*$ & \\
\hline Effects & Front & Central & Back & Severity & Diphthong & \\
\hline Estimate & 0.36 & 0.80 & 0.44 & -1.57 & 0.08 & \\
\hline Sign. & $* * *$ & $* * *$ & $* * *$ & $* * *$ & & \\
$\begin{array}{l}* * * \mathrm{p}<0.001, \\
* \mathrm{p}<0.05\end{array}$
\end{tabular}

baseline word lists. The recognition score of each word is defined as a weighted sum of the number of phonemes in each phoneme class counted from canonical pronunciation and the estimates of the Manner-Frontness model. The word error rate (WER) is the rate of the number of substitution and deletion errors from the total recognition number.

As shown in Table 2, the average WER of 100 trials of the random selection is $17.2 \%$. WERs using three alphabet word lists are $16.5 \sim 18.2 \%$. Using the maximum of the number of phonemes as criterion for selecting words shows a $16.8 \%$ rate and using the minimum criterion shows a $21.1 \%$ rate. The differences between the WERs of baselines are not significant.

Table 2. Recognition results

\begin{tabular}{|c|c|c|c|c|c|c|c|}
\hline \multirow{3}{*}{$\begin{array}{c}\text { WER, } \\
\%\end{array}$} & \multicolumn{6}{|c|}{ Baseline } & \multirow{2}{*}{$\begin{array}{c}\text { Recognition } \\
\text { Score }\end{array}$} \\
\cline { 2 - 6 } & \multicolumn{2}{|c|}{ Phonetic Alphabet } & Minimum & Maximum & RandomSelection & \\
\cline { 2 - 7 } & Set 1 & Set 2 & Set 3 & & 16.8 & 17.2 & 13.8 \\
\hline
\end{tabular}

Using the word list with the highest recognition score, a $13.8 \%$ WER is obtained, which is a $16.4 \%$ relative improvement compared to the baseline. The improvement is statistically significant $(\mathrm{p}<0.05)$.

\section{Conclusions}

In this paper, four sets of the articulation features for each word are defined and the relationship between the features and WERs is analyzed using a GLMM. Among the models, the Manner-Frontness model is selected as the best one by the analysis. Estimates of the GLMM are observed and applied to calculate the recognition score. The results from Table 1 that increasing the number of Fricative increases the recognition error rates and the number of Nasal increases the accuracy are consistent with the analysis of the articulation errors of the English dysarthric speech. The difference 
between monophthong and diphthong is also consistent. The fortis phonemes which are complex to articulate seem to lower the estimate of Plosive. The vocabulary is optimized with words having the maximum recognition score for voice keyboard interface and, as a result, the WER decreases by $16.4 \%$, relatively. The results would be extended to other language by the mapping between the class of the phoneme in Korean and other language.

Acknowledgements. This work has been supported by the R\&D Program of MKE/KEIT (10036461, Development of an embedded key-word spotting speech recognition system individually customized for disabled persons with dysarthria).

\section{References}

1. Rudzicz, F.: Adjusting dysarthric speech signals to be more intelligible. Comput. Speech Language 27(6), 1163-1177 (2013)

2. Christensen, H., Aniol, M.B., Bell, P., Green, P., Hain, T., King, S., Swietojanski, P.: Combining in-domain and out-of-domain speech data for automatic recognition of disor-dered speech. In: Proceedings of INTERSPEECH 2013, pp. 3642-3645 (2013)

3. Morales, S.O.C., Cox, S.J.: Modelling errors in automatic speech recognition for dy-sarthric speakers. EURASIP J. Adv. Signal Process. 2009, 308-340 (2009)

4. Hawley, M.S., Cunningham, S.P., Green, P.D., Enderby, P., Palmer, R., Sehgal, S., O'neill, P.: A voice-input voice-output communication aid for people with severe speech im-pairment. IEEE Trans. Neural Syst. Rehabil. Eng. 21(1), 23-31 (2013)

5. Kim, S., Hwang, Y., Shin, D., Yang, C.-Y., Lee, S.-Y., Kim, J., Kong, B., Chung, J., Cho, N., Kim, J.-H., Chung, M.: VUI development for Korean people with dysarthria. J. Assistive Technol. 7(3), 188-200 (2013)

6. Hamidi, F., Baljko, M., Livingston, N., Spalteholz, L.: CanSpeak: a customizable speech interface for people with dysarthric speech. In: Miesenberger, K., Klaus, J., Zagler, W., Karshmer, A. (eds.) ICCHP 2010, Part 1. LNCS, vol. 6179, pp. 605-612. Springer, Heidelberg (2010)

7. Platt, L.J., Andrews, G., Howie, P.M.: Dysarthria of adult cerebral palsy: II. phonemic analysis of articulation errors. J. Speech Language Hear. Res. 23, 41-55 (1980)

8. Whitehill, T.L., Ciocca, V.: Speech errors in Cantonese speaking adults with cerebral palsy. Clin. Linguist. Phonetics 14(2), 111-130 (2000)

9. Mengistu, K.T., Rudzicz, F.: Comparing humans and automatic speech recognition sys-tems in recognizing dysarthric speech. In: Advances in Artificial Intelligence, pp. 291-300 (2011)

10. Choi, D-L., Kim, B-W., Lee, Y-J., Um, Y., Chung, M.: Design and creation of dysarthric speech database for development of QoLT software technology. In: Proceedings of International Conference on Speech Database and Assessments 2011, pp. 47-50 (2011)

11. Povey, D., Ghoshal, A., Boulianne, G., Burget, L., Glembek, O., Goel, N., Hannemann, M., Motlicek, P., Qian, Y., Schwarz, P., Silovsky, J., Stemmer, G., Vesely, K: The kaldi speech recognition toolkit. In: Proceedings of ASRU 2011 (2011) 